\title{
Stealth Chaos due to Frame-Dragging
}

\author{
Andrés F. Gutierrez ${ }^{1}$ Alejandro Cárdenas-Avendaño ${ }^{2}{ }^{3}$, \\ Nicolás Yunes ${ }^{3}$ and Leonardo A. Pachón ${ }^{1}$ \\ 1 Grupo de Física Teórica y Matemática Aplicada, Instituto de Física, Facultad de \\ Ciencias Exactas y Naturales, Universidad de Antioquia, Medellín, Colombia. \\ 2 Programa de Matemática, Fundación Universitaria Konrad Lorenz, 110231 Bogotá, \\ Colombia. \\ 3 Illinois Center for Advanced Studies of the Universe, Department of Physics, \\ University of Illinois at Urbana-Champaign, Urbana, IL 61801, USA.
}

\begin{abstract}
.
While the geodesic motion around a Kerr black hole is fully integrable and therefore regular, numerical explorations of the phase-space around other spacetime configurations, with a different multipolar structure, have displayed chaotic features. In most of these solutions, the role of the purely general relativistic effect of frame-dragging in chaos has eluded a definite answer. In this work, we show that, when considering neutral test particles around a family of stationary axially-symmetric analytical exact solution to the Einstein-Maxwell field equations, frame-dragging (as captured through spacetime vorticity)is capable of reconstructing KAM-tori from initially highly chaotic configurations. We study this reconstruction by isolating the contribution of the spacetime vorticity scalar to the dynamics, and exemplify our findings by computing rotation curves and the dimensionality of phase-space. Since signatures of chaotic dynamics in gravitational waves have been suggested as a way to test general relativity in the strong-field regime, we have computed gravitational waveforms using the semirelativistic approximation and studied the frequency content of the gravitational wave spectrum. If this mechanism is generic, chaos suppression by frame-dragging may undermine present proposals to verify the hypothesis of the no-hair theorems and the validity of general relativity.
\end{abstract}




\section{Introduction}

One of the most studied sources of gravitational waves are extreme-mass-ratio inspirals (EMRIs), which consist of a low mass compact object, like a stellar black hole or a neutron star that inspirals into a supermassive black hole [1, 2, 3]. The gravitational waves emitted by these types of systems constitute a way to map the multipole structure of the spacetime, i.e., if the spacetime is stationary and axially-symmetric, the gravitational waves generated by a particle in a near-circular orbit, carry the multipole moment information of the central object $[4,5]$. If the central object is a Kerr black hole, the no-hair theorems $[6,7,8]$ for electrically-neutral, isolated black holes imply that all the multipole moments of the source are uniquely and fully characterized by the mass and the spin angular momentum of the source. Due to the particular features of the Kerr solution, evidence of black holes outside general relativity, containing higher-order independent multipole moments, would lead to a change of paradigm in gravitational physics $[9,10,11,12,13,14,15,16,17]$.

In terms of dynamical systems, the geodesic motion around a Kerr black hole is fully integrable and can be solved by the specification of four constants of motion, namely the rest mass of the orbiting body, the orbital energy, the orbital angular momentum and the Carter constant, $M, E, L$ and $Q$, respectively $[18,19,20]$. Under a Hamiltonian formulation of the geodesic motion, chaos refers to the non-integrability of the equations of motion, i.e., the non-existence of a smooth analytic function that interpolates between orbits [21]. This notion of integrability of Hamiltonian systems is known as Liouville integrability: if $n$ linearly independent integrals of motion exist in a system of $n$ degrees of freedom, then there exists a coordinate transformation to angle-action variables such that the equations of motion can be put in quadrature form [22].

According to the Kolmogorov-Arnold-Moser (KAM) theorem [23], small perturbations of an integrable system imply that some of the invariant tori are deformed and survive, while others are destroyed. These perturbations can arise from external sources (e.g., discs or rings [24, 25] surrounding the source) or due internal changes in the multipole structure of the source; the latter are the ones we explore in this work.

When the perturbation destroys the invariant tori, the resonant orbits in phase space disintegrate to form Birkhoff chains of islands [26], where the fundamental orbital frequencies are different for each member of the chain, although the ratio of these frequencies is the same for each curve [13]. In contrast, the variation of the frequency between consecutive orbits, which do not belong to a Birkhoff island or a chaotic layer $[22,13]$, is a monotonic function of the radial coordinate. This unique characteristic can be used as an indicator of extra multipole moments of the central source and the observation of a gravitational wave with a constant frequency ratio could imply the presence of a non-Kerr background [13].

Over the past years, several numerical explorations of the phase-space around astrophysical sources that have an independent quadrupole moment have shown the appearance of chaos in exact solutions to the Einstein field equations that have arbitrary 
multipole moments. For example, the Manko-Novikov [27] and the Pachon-RuedaSanabria-Gomez (PRS) [28, 29] solutions are axially symmetric configurations that guarantee that the mass $M, E$ and $L$ are constants of the motion but they do not possess an analogous Carter constant [30, 31, 32, 33], and therefore, geodesic chaos is expected.

With the future space-based detector Laser Interferometer Space Antenna (LISA) it may be possible to measure fractional deviations from the quadrupole moment of the Kerr solution at a sub-percent level of accuracy [9, 3]. Thus, depending on the mass ratio, there could be EMRIs that will slowly cross a resonance and, if the spacetime is not regular, leave a detectable signature of chaos [13].

In this paper, we show that, for neutral particles, the introduction of the purely general relativistic effect of frame-dragging, as parameterized through a vorticity scalar computed from the vorticity tensor of a congruence world-lines describing static observers [34], is capable of reconstructing the KAM tori from an initial highly chaotic configuration around a stationary gravitational source described by the PRS metric [28]. Given that the vorticity scalar encodes the precession frequency of a gyroscope attached to the world-lines of a static observer, this quantity is a good proxy for frame-dragging effects. If this effect is generic, frame-dragging may suppress chaotic signatures e.g., Birkhoff chains in EMRIs around non-Kerr objects, which would prevent probes of the assumptions of the no-hair theorems with gravitational waves.

The layout of this paper is as follows. In Sec. 2 we introduce the PRS metric and the figures of merit we use to display the presence of chaos. To exemplify the effect of rotationally induced multipole moments on the EMRI dynamics around a central compact object, in Secs. 3 and 4, respectively, we study the following two relevant scenarios: in KAM Tori Reconstruction I, we consider a stationary central object without an electromagnetic field; and in KAM Tori Reconstruction II, we consider a stationary central object with an electromagnetic field. In both of these examples, the orbiting neutral particle develops a mixture of regular and chaotic dynamics, but as the vorticiy of the spacetime is increased, the chaotic features are diminished. In Sec. 5 we provide a theoretical explanation of why chaos is being suppressed in this context. Finally, in Sec. 6, we present our discussion, ideas for future work and conclude. Throughout this paper we use geometric units with $c=1=G$.

\section{Theoretical Framework}

The emergence of chaos around astrophysical objects that deviate from the Kerr black hole is driven by the intricate interplay between strong field corrections from different multipole orders. Let us then consider the PRS metric, designed to describe the exterior gravitational field of a reflection-symmetric source, with generic mass monopole $M$, spin angular momentum per unit mass $a=J / M$, gravitational quadrupole moment $\mathcal{Q}^{\mathrm{G}}$, current octupole moment $\mathcal{S}^{\mathrm{G}}$ and mass hexadecapole moment $\mathcal{H}^{\mathrm{G}}$, along the symmetry axis. This solution is given in terms of the quasi-cylindrical Weyl-Lewis-Papapetrou 
coordinates $x^{\mu}=(t, \rho, z, \phi)$ as

$$
\mathrm{d} s^{2}=-f(\mathrm{~d} t-\omega \mathrm{d} \phi)^{2}+f^{-1}\left[e^{2 \gamma}\left(\mathrm{d} \rho^{2}+\mathrm{d} z^{2}\right)+\rho^{2} \mathrm{~d} \phi^{2}\right],
$$

where the metric functions $f(\rho, z), \omega(\rho, z)$ and $\gamma(\rho, z)$ are obtained from the Ernst complex potentials $\mathcal{E}(\rho, z)$ and $\Phi(\rho, z)$, which obey the following relations [35]

$$
\begin{aligned}
\left(\Re[\mathcal{E}]+|\Phi|^{2}\right) \nabla^{2} \mathcal{E} & =\left(\nabla \mathcal{E}+2 \Phi^{*} \nabla \Phi\right) \cdot \nabla \mathcal{E}, \\
\left(\Re[\mathcal{E}]+|\Phi|^{2}\right) \nabla^{2} \Phi & =\left(\nabla \mathcal{E}+2 \Phi^{*} \nabla \Phi\right) \cdot \nabla \Phi .
\end{aligned}
$$

The Ernst equations (2) can be solved by means of the Sibgatullin's integral method [36], according to which the complex potentials $\mathcal{E}$ and $\Phi$ can be calculated from specified axis data $\mathcal{E}(z, \rho=0)$ and $\Phi(z, \rho=0)$. The details and the explicit solution can be found in Refs. [28, 29]. Here we will only show the multipole moments, which are [28]

$$
\begin{aligned}
& \mathcal{Q}^{\mathrm{G}}=M\left(k-a^{2}\right), \quad \mathcal{S}^{\mathrm{G}}=-M a\left(a^{2}-2 k\right), \\
& \mathcal{H}^{\mathrm{G}}=\frac{1}{70} M\left[70 a^{4}-210 a^{2} k+13 a q \mu+10 k\left(7 k-M^{2}+q^{2}\right)+3 \mu^{2}\right] .
\end{aligned}
$$

The parameter $k$ allows the source to have an arbitrary mass quadrupole moment, without changing its mass or spin angular momentum, while the magnetic dipole parameter $\mu$ changes the mass hexadecapole moment, without changing any other lower multipole. In the electromagnetic sector, the solution has a magnetic dipole moment $\mathcal{B}^{\mathrm{E} \& \mathrm{M}}=\mu+a q$ and an electric quadruple moment $\mathcal{Q}^{\mathrm{E} \& \mathrm{M}}=-a^{2} q-a \mu+k q$, where $q$ is the electric charge. The electromagnetic field is then fully determined by the charge $q$, the parameter $\mu$, the dimensional spin angular momentum $a$ and the gravitational mass quadrupole parameter $k$. Observe that one can have an electric field even when $q=0$ that is induced by rotation, i.e., provided $\mu$ and $a$ are non-vanishing [37]. The PRS metric reduces to the Kerr-Newman solution when $k=0$ and $\mu=0$.

In the PRS solution, the vorticity scalar of spacetime $\omega_{\mathrm{v}}$ originates from a combination of the mass currents and the non-vanishing Poynting vector of the electromagnetic field, which can be written as [29]

$$
\omega_{\mathrm{v}}=\sqrt{\omega^{\alpha}{ }_{\beta} \omega_{\alpha}{ }^{\beta}}=\frac{e^{-\gamma}}{\sqrt{2 f}} \sqrt{\Im\left[\mathcal{E}_{, z}+2 \Phi^{*} \Phi_{, z}\right]^{2}+\Im\left[\mathcal{E}_{, \rho}+2 \Phi^{*} \Phi_{, \rho}\right]^{2}},
$$

where the vorticity tensor is defined as $\omega_{\alpha \beta}=u_{[\alpha ; \beta]}+\dot{u}_{[\alpha} u_{\beta]}$ for a congruence of static observers with tangent vector $u^{\alpha}[38]$. The vorticity scalar is a function of global quantities of the spacetime, such us the spin angular momentum, deformation parameters, or the charge for the electromagnetic case. One can show, as done by Rindler and others in the 1990s [39], that the vorticity scalar is directly proportional to the rate of precession

of gyroscopes outside of a massive source. It is in this sense that the vorticity scalar encodes frame dragging effects.

\subsection{Figures of merit: the rotation number and the correlation dimension}

We quantitatively study the possible reconstruction of KAM tori due to frame-dragging through rotation curves $\nu_{\theta}[22]$ and the dimensionality $D$ of phase space through the 
correlation dimension $C(\varrho)[40]$. The rotation curve $\nu_{\theta}$ provides information on the localization and emergence of Birkhoff islands, while the correlation dimension encodes the dimensionality of the manifold in which the initial condition is allowed to evolve by considering correlations between points of a long-time series on the manifold [40].

The rotation number is defined as [22]

$$
\nu(\rho)=\lim _{N \rightarrow \infty} \frac{1}{2 \pi N} \sum_{i=1}^{N} \theta_{i},
$$

where $\theta_{i}$ is the clockwise angle subtended by two vectors, defined from the invariant point, $(\rho, \dot{\rho})$, to two consecutive successive piercings of the Poincare's surface of section, i.e., $\theta_{i}=\measuredangle\left(\vec{v}_{i+1}, \vec{v}_{i}\right)$ [22]. The rotation curve of the system is obtained by evaluating the rotation number as a function of the location of the Poincaré surface of section in phase space, which for this work we define as the location of the surface by the minimum value of the radial coordinate sampled by that surface. Abrupt changes in the rotation curve signal the presence of chaotic orbits $[22,13]$.

The chaotic features displayed in the rotation curve can sometimes be hard to discern by naked eye, due to subtle changes in the monotonicity of the curve. As an alternative, it is possible to focus on the dimension of the invariant manifold in which the motion takes place, as conservative chaotic systems have an integer dimension of the invariant manifolds, whereas the dissipative have a non-integer dimension [41].

Among different proposals for measuring the invariant-manifold dimension [41], we focused on the correlation dimension introduced by Grassberger \& Procaccia in Ref. [40]. In this approach, the phase-space distance $\varrho \ll 1$ and the correlation dimension $C(\varrho)$ is proportional to $\varrho^{D}$, where $D$ is the dimensionality of of the phase-space orbit. In operational terms, $C(\varrho)$ is calculated as follows. Given $N$ sampled points on the orbit, the correlation dimension is defined as [42]

$$
C(\varrho)=\lim _{N \rightarrow \infty} \frac{1}{N^{2}} \sum_{i=1}^{N} \sum_{j=1, j \neq i}^{N} \Theta\left(\varrho-\left|\xi_{i}-\xi_{j}\right|\right),
$$

where $\xi_{i}=(\rho, \dot{\rho}, z, \dot{z})$ represents a phase-space vector and $\Theta$ is the Heaviside step function. Orbits with $D=1$ are close to the invariant point or to small Bifkhoff islands that surround periodic orbits, while orbits with $D=2$ or 3 are integrable [41].

With the background defined and these two tools to study the dynamics, we now follow the procedure described in Ref. [16] to obtain the geodesic motion in the PRS metric. We numerically integrate the Hamiltonian equations of motion for the variables $\dot{\rho}, \dot{z}, \dot{P}_{\rho}, \dot{P}_{z}$, using an explicit Runge-Kutta method due to Dormand and Prince [43]. We have checked that the conserved quantities remain constant, e.g., errors did not exceed the order of $\Delta E / E=10^{-12}$, under the numerical evolution, ensuring that the particle does not wander in phase space due to numerical error. 


\section{KAM Tori Reconstruction I}

Consider, as an example, a stationary compact object described by the PRS metric [28, 29] with mass $M$, mass quadrupole moment $\mathcal{Q}^{\mathrm{G}}=1.6 M^{3}$, and $j=0=\mu$, implying $k \neq 0$. Here we have introduced the dimensionless spin parameter $j \equiv a / M$. Since $\mathcal{Q}^{\mathrm{G}}>0$ when $j=0$, the source is prolate with a significant deviation from the Kerr intrinsic oblate deformation, and therefore, the corresponding phase space will suffer a substantial deviation from integrability, and one expects chaotic dynamics [14].

The top-left panel in Fig. 1 shows the Poincaré surfaces of section for a test particle moving on this background with $E=0.95$ and $L=3 \mathrm{M}$. The main islands in the center of the figure are surrounded by a chaotic sea of layers with many high-multiplicity islands of stability, caused by geodesics that visit the same equilibrium point multiple times. This strong chaos is also manifest in the associated dimensionality of phase space and the rotation curves, shown in the middle and bottom panels of the figure, respectively. Observe how in the shaded regions, $\nu_{\theta}$ shows non-monotonic variations and a large plateau, all of which are clear signatures of a non-integrable system.
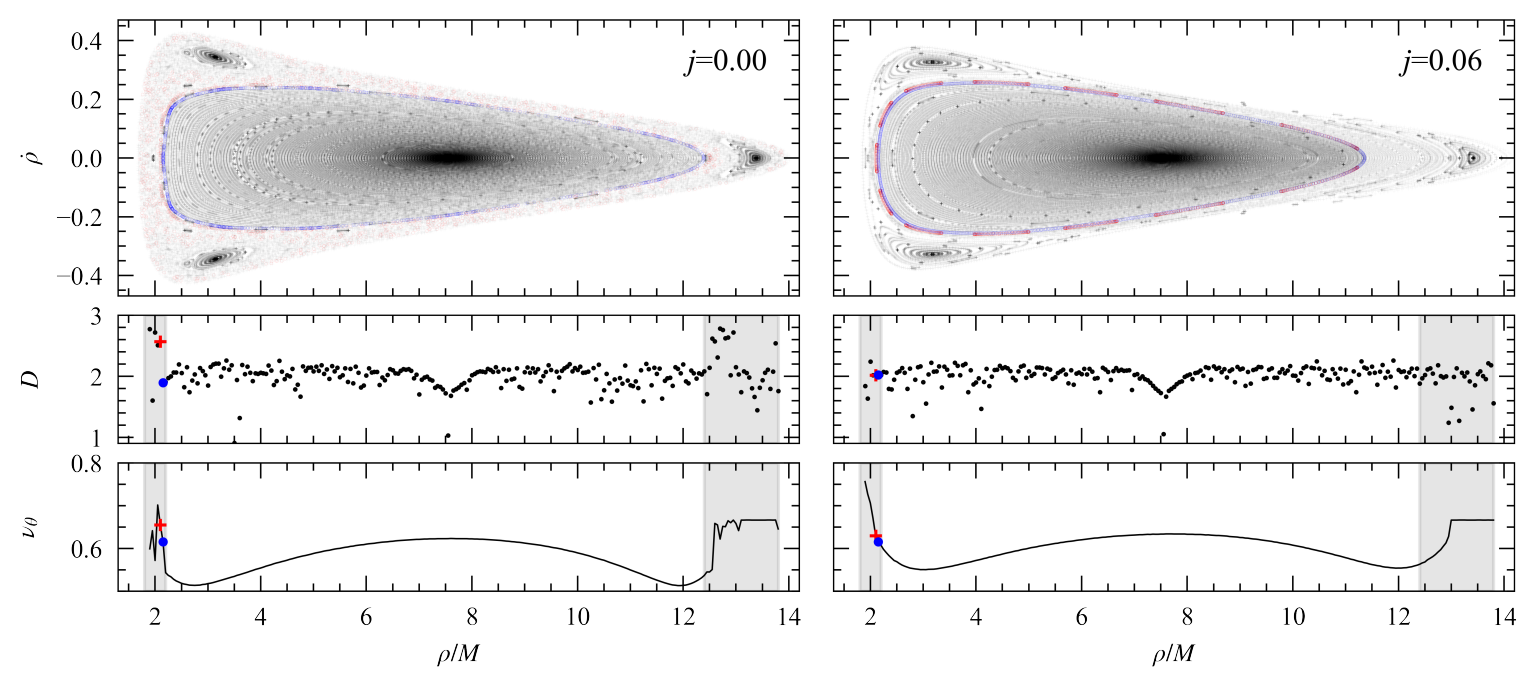

Figure 1. Poincaré surfaces of section (top), the dimensionality of the phase-space orbits (middle), and the rotation curves (bottom), for orbits with $E=0.95, L=3 \mathrm{M}$ and $\mathcal{Q}^{\mathrm{G}}=1.6 \mathrm{M}^{3}$. The left panels correspond to a background with zero dimensionless spin parameter $j=0.0$, while the right panels have $j=0.06$, while maintaining $\mathcal{Q}^{\mathrm{G}}$ constant. The increase in the dimensionless spin parameter decreases the chaotic structure observed in the rotation curves and in the dimensionality, especially in the shaded regions. The two middle and lower panels also highlight the two particular initial conditions (one at $\rho=2.10 \mathrm{M}$ (red) and one at $\rho=2.15 \mathrm{M}$ (blue)) for which we will calculate gravitational waves in the semi-relativistic approximation.

Let us now endow the spacetime with vorticity, and therefore introduce framedragging effects, by increasing the spin angular momentum of the source to a fixed number, while keeping $\mu=0=q$, and varying $k$ in Eq. (3) in such a way that $\mathcal{Q}^{\mathrm{G}}$ 
remains constant. We find that when doing so, the integrability of the orbits increases, in the sense that the rotation number becomes more regular and the dimensionality of the phase-space manifold goes down to two. The right panels of Fig. 1 show this result for orbits with the same initial conditions as the left panel, but with $j=0.06$ (equivalent to an angular rotation frequency of the horizon of a few times $10^{-3} \mathrm{~Hz}$ for a $10^{6} M_{\odot}$ $\mathrm{BH}$ ). The rapid non-monotonic variations in the shaded regions shown in Fig. 1 when $j=0$ (left panel) disappear when $j=0.06$ (right panel), and the integrable orbits get grouped in a band around $D(\rho) \approx 2$, which indicates they are becoming confined to a 2-dimensional torus. The results presented here are examples of the characteristic of what we find in numerical experiments over a vast region of the parameter space that represent different source configurations and initial conditions.

\subsection{Gravitational waves in the semi-relativistic approximation}

While the appearance of the features highlighted in Fig. 1 are considered as generic chaotic signatures, these quantities are not physical observables. As the non-integrability of a Hamiltonian system changes the evolution of the fundamental frequencies of the orbital motion $[44,13]$, the islands of instability and prolonged resonant regimes, shown in Fig. 1, must be encoded directly in the gravitational waves emitted [3] by these systems.

To show how chaos may affect the gravitational waves emitted, we follow Ref. [11] and calculate approximate gravitational waveforms using the semi-relativistic approximation [45], i.e., neglecting gravitational wave dissipation and the conservative selfforce. This semi-relativistic "kludges" are widely used to explore generic EMRIs [46, 47].

We have evolved two nearby orbits, $\rho_{i}=2.10 M$ (denoted in red in Fig. 1) and $\rho_{i}=2.15 M$ (denoted in blue in Fig. 1) and computed the approximate gravitational waves. In Fig. 2 we show the "plus" polarization, $h_{+}$, of these two initial conditions for $j=0.0$. While these waveforms are very rich in structure, due to the eccentricity and inclination of the orbits, both features expected for some EMRIs that will radiate in the LISA band [2], the chaotic features are not manifest in the time domain.

In the frequency domain, however, the separation between regular and chaotic conditions is more clear. In Fig. 3 we show the Fourier transform of these approximate waveforms. Observe that when $j=0.0$, (left panel), the chaotic trajectories induce a multi-peak structure on the Fourier transform of the emitted gravitational waves, while this structure is absent when $j=0.06$ (right panel). The difference between both spectra is now evident: gravitational waves from chaotic configurations are denser in frequency content than gravitational waves from regular configurations, as a result of the very nature of chaos. In the presence of frame-dragging, $j \neq 0$, these "extra" frequency components induced by chaos are suppressed and the spectra becomes simpler.

For sufficiently slow evolutions, i.e., small-mass ratios, this richness and structure of the frequency components may have a measurable impact in the gravitational waveform [13]. As we chose the source parameters for the approximate gravitational 


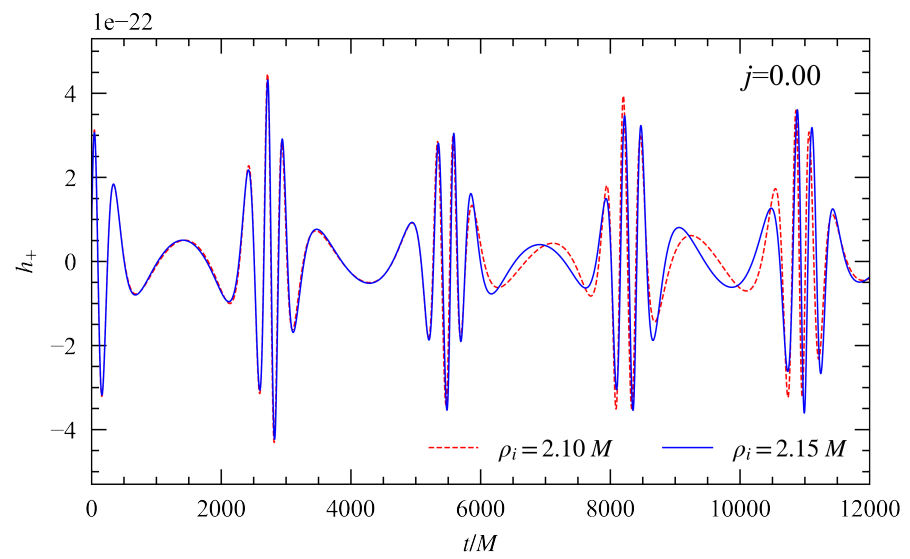

Figure 2. Approximate waveforms $\left(h_{+}\right)$produced by a $20 M_{\odot}-10^{6} M_{\odot}$ at $4 \mathrm{Gpc}$, observed at polar angle $\pi / 6$ and azimuthal angle $\pi / 4$, for $j=0.0$, for two nearby initial conditions, i.e., at $\rho_{i}=2.1 M$ (red dashed lines) and at $\rho_{i}=2.15 M$ (blue solid lines). Other orbital parameters are the same ones as those used in Fig. (1), i.e., $E=0.95$ and $L=3.0 M$ and $\mathcal{Q}^{\mathrm{G}}=1.6 \mathrm{M}^{3}$. As it can be seen, the waveforms slowly start to differ given the difference in the initial conditions, and chaos is not easily manifest in the time domain.

waveforms to fall well within the LISA band [2], for an astrophysical source this would imply that detecting chaos may be more difficult than previously anticipated due to the stealth effect that frame-dragging has.
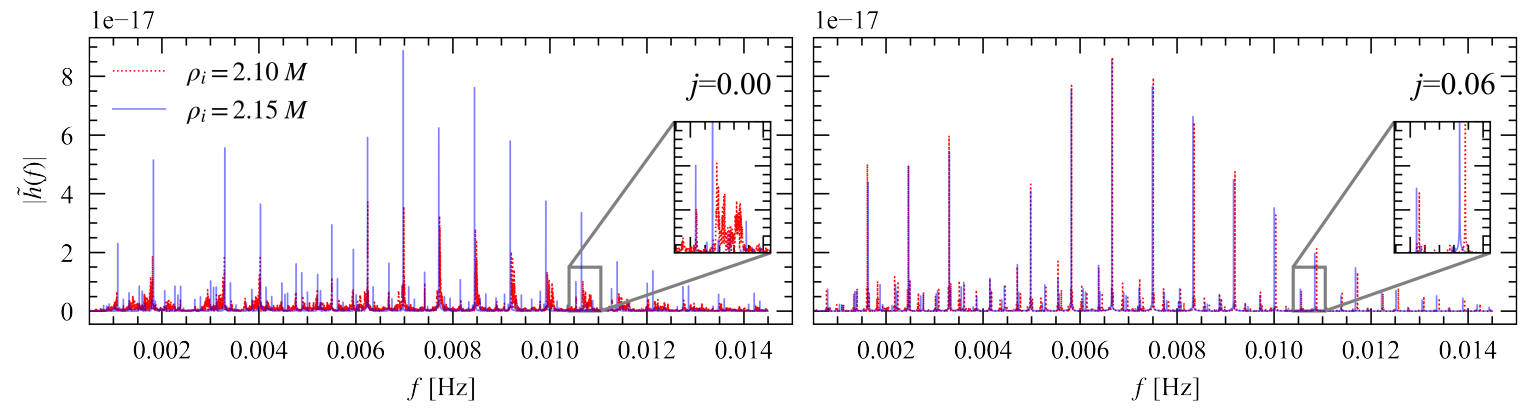

Figure 3. Absolute value of the Fourier transform of $h=h_{+}+i h_{\times}$produced by a $20 M_{\odot}-10^{6} M_{\odot}$ at $4 \mathrm{Gpc}$, observed at polar angle $\pi / 6$ and azimuthal angle $\pi / 4$, for $j=0$ (left panel) and $j=0.06$ (right panel), for two very close initial conditions, i.e., at $\rho_{i}=2.1 M$ (red dashed lines) and at $\rho_{i}=2.15 M$ (blue solid lines). Other orbital parameters are the same ones as those used in Fig. (1), i.e., $E=0.95$ and $L=3.0 \mathrm{M}$ and $\mathcal{Q}^{\mathrm{G}}=1.6 \mathrm{M}^{3}$. Orbital chaos manifests itself through a multi-peak structure in the Fourier amplitude of gravitational waves, as seen in the inset of the left panel. When the spin-parameter is increased, however, this multi-peak structure is suppressed, and thus suggesting that the chaotic-to-regular dynamics transition may be addressed in terms of the frequency content of the spectrum. 


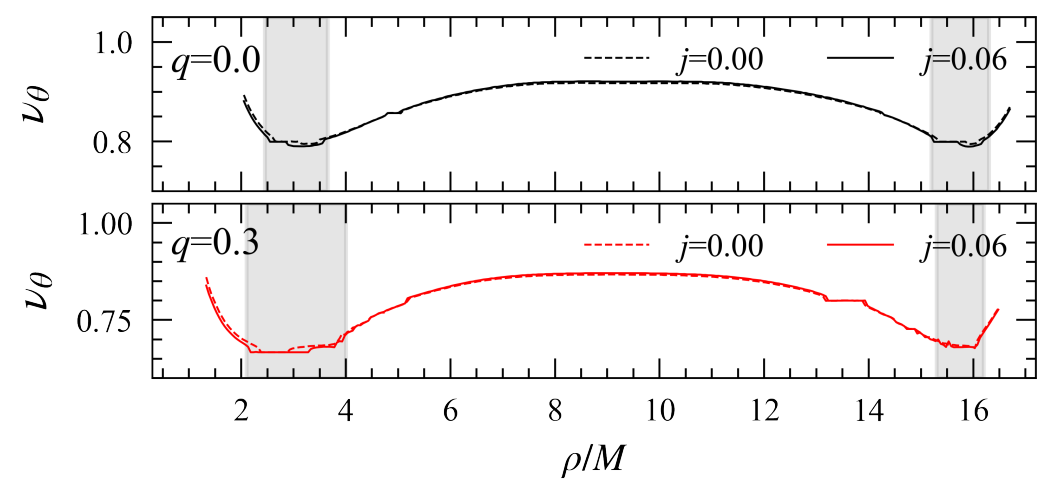

Figure 4. Rotation curves for an orbit with $E=0.95$ and $L=2.30 M$ in a background with $\mathcal{Q}^{\mathrm{G}}=0, \mu=15 M^{2}$, and $q=0.0$ (top) or $q=0.3$ (bottom), and with dimensionless spin parameter $j=0.0$ (dashed lines) and $j=0.06$ (solid lines), while maintaining $\mathcal{Q}^{\mathrm{G}}$ constant. As in the previous cases, an increase in the spin parameter generates a smooth transition to regular dynamics. In the charged case, however, spin is not as effective as before at suppressing chaos.

\section{KAM Tori Reconstruction II}

Let us now study a different example in which the spacetime has an electromagnetic contribution. Let us consider a test particle in an orbit with $E=0.95$ and $L=2.3 \mathrm{M}$ in a PRS spacetime [28], with mass $M$, vanishing quadrupole $\mathcal{Q}^{\mathrm{G}}=0$ and a magnetic dipole $\mu=15 M^{2}$. The electromagnetic multipole moments now break the integrability of the system, as shown in the shaded regions of the top panel of Fig. 4 by the non-monotonic behavior of the rotation curve. This is not because of the mass quadrupole moment, which is zero here, but rather due to energy density of electromagnetic field sourcing a non-trivial mass octupole moment $\mathcal{H}^{\mathrm{G}}$ [see Eq. (3)]. As in the previous case, when the spin parameter is increased from $j=0.0$ to $j=0.06$, the chaotic behavior is suppressed, but this time the suppression is not as effective as in the not magnetized case. This is shown in the top panel of Fig. 4 by the length of the plateaus, corresponding to the resonant KAM tori, decreasing in the rotating case.

For rotating sources without electromagnetic fields $(\Phi=0$, as explored in the previous section), $\Im[\mathcal{E}] \neq 0$ and provides the standard contribution of the spin angular momentum to the vorticity scalar of the spacetime. On the other hand, in the presence of non-aligned electric and magnetic fields, the terms $\Phi^{*} \Phi_{, z}$ and $\Phi^{*} \Phi_{, \rho}$ are non-zero, and therefore the spacetime is endowed with a non-vanishing vorticity scalar. The contribution of the electromagnetic fields means that one can generate spacetime vorticity when $q \mu \neq 0$, even if the central object is not spinning, as originally suggested by Bonnor [48], and later explained in Ref. [37].

The fact that the vorticity scalar can also be sourced through the electromagetic field, naturally allows for a new scenario to study the suppression of chaos. However, the presence of an electric charge monopole also induces a prolate contribution to quadrupole deformation, which tends to generate chaotic dynamics [49]. The bottom panel of Fig. 4 
shows how the electric charge of the source creates new chaotic zones, but as spin is increased from $j=0.0$ to $j=0.06$, these chaotic structure is not suppressed as efficiently as in the previous section.

\section{Is the vorticity of the spacetime suppressing chaos in the PRS metric?}

To understand the behavior displayed in the examples shown in the previous two sections, let us consider the complex Ernst potential $\xi$, which has a direct relation to the Newtonian gravitational potential $[35,50,49]$ and from which the multipole moments in Eq. (3) were derived [50]. By performing a Taylor expansion of $\xi(\rho, z)$ in spherical coordinates $(r, \theta)$ at spatial infinity, its real $\Re[\xi]$ and imaginary $\Im[\xi]$ parts are

$$
\begin{aligned}
& \Re[\xi] \approx-\frac{M}{r}-\frac{\mathcal{Q}^{\mathrm{G}}}{r^{3}} P_{2}(\cos \theta)+O\left(r^{-5}\right), \\
& \Im[\xi] \approx \frac{a M}{r^{2}} P_{1}(\cos \theta)+O\left(r^{-4}\right),
\end{aligned}
$$

where $r=\sqrt{\rho^{2}+z^{2}}, \cos \theta=z / r$ and $P_{n}$ denotes the Legendre polynomial of $n$-th degree. The real part of the Ernst potential provides information about the Newtonian gravitational potential [50,49], which contains contributions from the mass monopole and the mass quadruple deformation $[51,14]$.

Given that Newtonian orbits for two test-particles (with zero quadrupole moment) are integrable, it is the quadrupole moment that could break the integrability of the systems [51, 14]. The imaginary part of the Ernst potential, on the other hand, contains the leading-order contribution of the rotation of the source to the gravitational field, and therefore it is responsible for the reconstruction of the broken tori. This is because this term changes the effective potential barrier for the orbiting particles, which we have kept fixed by not changing $E$ and $L$, so that the contribution of the quadrupole moment deformation, which is what sources chaos, becomes effectively weaker. Due to the analytical control of the multipole structure of the source that we have in this particular spacetime, we are able to uniquely vary the magnitude of the vorticity scalar (and thus, the amount of frame-dragging) while keeping the mass multipoles $M$ and $\mathcal{Q}^{\mathrm{G}}$ constant, regardless of the value of the angular momentum of the source. Thus, the larger the spin angular momentum, the stronger the contribution of this term to the dynamics and therefore, the smaller the deviations from the Kerr spacetime.

Given that deviations from the Kerr solutions are not unique, increasing the vorticity scalar of the spacetime may not always display this suppression, because of other more dominant effects. For example, the fact that the suppression of chaos in the electromagnectic case is less effective happens because the electric charge monopole plays two roles simultaneously: it induces a spacetime vorticity scalar (and therefore frame-dragging), which suppresses chaos, but at the same time, it also induces a more prolate deformation, which sources chaos. Our numerical explorations indicate that the suppression effect is smaller than the sourcing of chaos, and that overall, vorticity-induced chaos suppression is less effective when the source is charged than in the uncharged case. 


\section{Concluding remarks}

We have investigated extreme mass-ratio inspirals in the PRS bumpy spacetime through a test-particle approximation and found a novel mechanism, i.e., frame-dragging-assisted suppression of chaos. The analytical control of the multipole structure of the PRS metric, allowed us to introduce frame-dragging effects while keeping the quadrupole moment $\mathcal{Q}^{\mathrm{G}}$ of the source fixed, and see its effect to the dynamics of the motion of the test particles. Given that the quadrupole moment is responsible for the break of the integrability of the system, frame dragging (as quantified through the vorticity scalar) increases the regularity of the orbits by making its contribution weaker.

A natural extension of the work presented here is towards studying the motion of charged particles, albeit not in the context of EMRIs. When considering charged particles, previous works have not found any clear and unique indication of the spin dependence with chaos (see for instance, Refs. $[52,53]$ ). Note that these results are not in conflict with our findings, as we are considering here neutral test particles and showed explicitly how the spin contribution of the source affects the multipole structure of the source and interacts with the particle. This effect may also be presented in such cases, but perhaps subdominant with respect to electromagnetic forces, and therefore not easily detectable.

As gravitational waves probe both the conservative (time-symmetric) and the dissipative (time-asymmetric) sectors of the gravitational theory, neglecting radiation reaction is only valid as a first approximation towards the understanding of the characterization of chaotic features with EMRIs. Despite recent impressive calculations from black-hole perturbation theory (see, for instance, Ref. [54]), the EMRI modeling within GR is still not finished. This makes the addition of a consistent radiation reaction for this setup out of the scope of this work.

The suppression discussed in this work implies that, although chaos may be technically present in the orbital motion and the gravitational waves emitted by extreme mass-ratio inspirals around non-Kerr central objects, it may be hidden by frame-dragging effects. If chaos operates in a stealth mode for orbits around spinning objects, its detectability (or constraints on its existence) may be challenging with future gravitational wave observations.

\section{Acknowledgments}

We thank the referees for their feedback, which enabled us to improve the presentation of our results and manuscript. A.F.G.-R. and L.A.P acknowledge financial support through Banco de la República grant 201926 (Project 4345). A.C.-A. and N.Y. acknowledge financial support through NASA grant No. NNX16AB98G, No. 80NSSC17M0041, No. 80NSSC18K1352 and NSF grant PHY-1759615. A.C.-A. also acknowledges funding from the Fundación Universitaria Konrad Lorenz (Project 5INV1). Computational efforts were performed on the Illinois Campus Cluster, a computing resource that is operated by 
the Illinois Campus Cluster Program (ICCP) in conjunction with the National Center for Supercomputing Applications (NCSA) and which is supported by funds from the University of Illinois at Urbana-Champaign.

\section{References}

[1] Babak S, Gair J, Sesana A, Barausse E, Sopuerta C F, Berry C P L, Berti E, Amaro-Seoane P, Petiteau A and Klein A 2017 Phys. Rev. D95 103012 (Preprint 1703.09722)

[2] Gair J R, Babak S, Sesana A, Amaro-Seoane P, Barausse E, Berry C P L, Berti E and Sopuerta C 2017 J. Phys. Conf. Ser. 840012021 (Preprint 1704.00009)

[3] Barausse E et al. 2020 (Preprint 2001.09793)

[4] Ryan F 1995 Phys. Rev. D 52 5707-5718

[5] Ryan F D 1996 Phys. Rev. D 53 3064-3069 (Preprint gr-qc/9511062)

[6] Israel W 1966 Nuovo Cim. B 44S10 1 [Erratum: Nuovo Cim.B 48, 463 (1967)]

[7] Carter B 1971 Phys. Rev. Lett. 26 331-333

[8] Hawking S 1972 Commun. Math. Phys. 25 152-166

[9] Barack L and Cutler C 2007 Phys. Rev. D75 042003 (Preprint gr-qc/0612029)

[10] Barausse E, Rezzolla L, Petroff D and Ansorg M 2007 Phys. Rev. D75 064026 (Preprint gr-qc/0612123)

[11] Gair J R, Li C and Mandel I 2008 Phys. Rev. D 77024035 (Preprint 0708.0628)

[12] Barausse E and Sotiriou T P 2008 Phys. Rev. Lett. 101099001 (Preprint 0803.3433)

[13] Apostolatos T A, Lukes-Gerakopoulos G and Contopoulos G 2009 Phys. Rev. Lett. 103111101 (Preprint 0906.0093)

[14] Letelier P S, Ramos-Caro J and López-Suspes F 2011 Physics Letters A 3753655 - 3658 ISSN 0375-9601 URL http://www.sciencedirect.com/science/article/pii/S0375960111010395

[15] Brink J, Geyer M and Hinderer T 2015 Phys. Rev. Lett. 114081102 (Preprint 1304.0330)

[16] Cárdenas-Avendaño A, Gutierrez A F, Pachón L A and Yunes N 2018 Class. Quant. Grav. 35 165010 (Preprint 1804.04002)

[17] Datta S and Bose S 2019 Phys. Rev. D 99084001 (Preprint 1902.01723)

[18] Carter B 1968 Commun. Math. Phys. 10 280-310

[19] Schmidt W 2002 Class. Quant. Grav. 192743 (Preprint gr-qc/0202090)

[20] Teukolsky S A 2015 Class. Quant. Grav. 32124006 (Preprint 1410.2130)

[21] Levin J 2006 Phys. Rev. D $\mathbf{7 4} 124027$ (Preprint gr-qc/0612003)

[22] Contopoulos G 2002 Astronomy and Astrophysics Library ISSN 0941-7834 URL http://dx.doi. org/10.1007/978-3-662-04917-4

[23] Tabor M 1989 Chaos and Integrability in Nonlinear Dynamics: An Introduction, by M. Tabor. Wiley Interscience, 1989

[24] Semerak O and Sukova P 2010 Mon. Not. Roy. Astron. Soc. 404 545-574 (Preprint 1211.4106)

[25] Polcar L, Suková P and Semerák O 2019 Astrophys. J. 87716 (Preprint 1905.07646)

[26] Rasband S N 2015 Chaotic dynamics of nonlinear systems (Courier Dover Publications)

[27] Manko V S and Novikov I D 1992 Classical and Quantum Gravity 9 2477-2487

[28] Pachon L A, Rueda J A and Sanabria-Gomez J D 2006 Phys. Rev. D 73104038 (Preprint gr-qc/0606060)

[29] Gutiérrez-Ruiz A F and Pachón L A 2015 Phys. Rev. D 91124047 (Preprint 1504.01763)

[30] Brink J 2008 Phys. Rev. D 78102002 (Preprint 0807.1179)

[31] Han W b 2008 Phys. Rev. D 77123007 (Preprint 1006.2234)

[32] Brink J 2010 Phys. Rev. D 81022001 (Preprint 0911.1589)

[33] Contopoulos G, Lukes-Gerakopoulos G and Apostolatos T 2011 Int. J. Bifurc. Chaos 21 2261-2277 (Preprint 1108.5057) 
[34] Misner C W, Thorne K and Wheeler J 1973 Gravitation (San Francisco: W. H. Freeman) ISBN 978-0-7167-0344-0, 978-0-691-17779-3

[35] Ernst F J 1968 Phys. Rev. 168 1415-1417

[36] Manko V S and Sibgatullin N R 1993 Classical and Quantum Gravity 10 1383-1404 URL https://doi.org/10.1088/0264-9381/10/7/014

[37] Herrera L, Gonzalez G, Pachon L and Rueda J 2006 Class. Quant. Grav. 23 2395-2408 (Preprint gr-qc/0602040)

[38] Herrera L and Hernandez Pastora J L 2000 Class. Quant. Grav. 17 3617-3626 (Preprint gr-qc/0006080)

[39] Rindler W and Perlick V 1990 General Relativity and Gravitation 22 1067-1081 URL https: //doi.org/10.1007/BF00757816

[40] Grassberger P and Procaccia I 1983 Phys. Rev. Lett. 50 346-349

[41] Lakshmanan M and Rajasekar S 2003 Characterization of Regular and Chaotic Motions (Berlin, Heidelberg: Springer Berlin Heidelberg) pp 235-258 ISBN 978-3-642-55688-3 URL https: //doi.org/10.1007/978-3-642-55688-3_8

[42] Barnes E I 2001 The Astrophysical Journal 559 736-753 ISSN 1538-4357 URL http://dx.doi. org/10.1086/322376

[43] Dormand J and Prince P 1980 Journal of Computational and Applied Mathematics 619 - 26 ISSN 0377-0427 URL http://www.sciencedirect.com/science/article/pii/0771050X80900133

[44] Kiuchi K and Maeda K i 2004 Phys. Rev. D70 064036 (Preprint gr-qc/0404124)

[45] Babak S, Fang H, Gair J R, Glampedakis K and Hughes S A 2007 Phys. Rev. D 75024005 [Erratum: Phys.Rev.D 77, 04990 (2008)] (Preprint gr-qc/0607007)

[46] Chua A J K, Moore C J and Gair J R 2017 Phys. Rev. D 96044005 (Preprint 1705.04259)

[47] Chua A J K, Katz M L, Warburton N and Hughes S A 2021 Phys. Rev. Lett. 126051102 (Preprint 2008.06071)

[48] Bonnor W 1991 Physics Letters A 15823 - 26 ISSN 0375-9601 URL http://www.sciencedirect. com/science/article/pii/0375960191903345

[49] Pachon L A and Dubeibe F L 2011 Class. Quant. Grav. 28055002 (Preprint 1009.2214)

[50] Sotiriou T P and Apostolatos T A 2004 Class. Quant. Grav. 21 5727-5733 (Preprint gr-qc/ 0407064)

[51] Guéron E and Letelier P S 2001 Phys. Rev. E 63(3) 035201 URL https://link.aps.org/doi/ 10.1103/PhysRevE.63.035201

[52] Takahashi M and Koyama H 2009 Astrophys. J. 693 472-485 (Preprint 0807.0277)

[53] Kopáček O, Karas V, Kovář J and Stuchlík Z 2010 The Astrophysical Journal 722 1240-1259 URL https://doi.org/10.1088\%2F0004-637x\%2F722\%2F2\%2F1240

[54] Pound A, Wardell B, Warburton N and Miller J 2020 Phys. Rev. Lett. 124021101 (Preprint 1908.07419) 